\title{
Granulocyte colony-stimulating factors for febrile neutropenia prophylaxis following chemotherapy: systematic review and meta-analysis
}

\author{
Katy L Cooper ${ }^{1 *}$, Jason Madan², Sophie Whyte ${ }^{1}$, Matt D Stevenson ${ }^{1}$ and Ron L Akehurst ${ }^{1}$
}

\begin{abstract}
Background: Febrile neutropenia (FN) occurs following myelosuppressive chemotherapy and is associated with morbidity, mortality, costs, and chemotherapy reductions and delays. Granulocyte colony-stimulating factors (GCSFs) stimulate neutrophil production and may reduce FN incidence when given prophylactically following chemotherapy.

Methods: A systematic review and meta-analysis assessed the effectiveness of G-CSFs (pegfilgrastim, filgrastim or lenograstim) in reducing FN incidence in adults undergoing chemotherapy for solid tumours or lymphoma. G-CSFs were compared with no primary G-CSF prophylaxis and with one another. Nine databases were searched in December 2009. Meta-analysis used a random effects model due to heterogeneity.

Results: Twenty studies compared primary G-CSF prophylaxis with no primary G-CSF prophylaxis: five studies of pegfilgrastim; ten of filgrastim; and five of lenograstim. All three G-CSFs significantly reduced FN incidence, with relative risks of 0.30 ( $95 \%$ Cl: 0.14 to 0.65 ) for pegfilgrastim, 0.57 (95\% Cl: 0.48 to 0.69) for filgrastim, and 0.62 (95\% $\mathrm{Cl}: 0.44$ to 0.88$)$ for lenograstim. Overall, the relative risk of FN for any primary G-CSF prophylaxis versus no primary G-CSF prophylaxis was 0.51 (95\% Cl: 0.41 to 0.62 ). In terms of comparisons between different G-CSFs, five studies compared pegfilgrastim with filgrastim. FN incidence was significantly lower for pegfilgrastim than filgrastim, with a relative risk of 0.66 (95\% Cl: 0.44 to 0.98$)$.
\end{abstract}

Conclusions: Primary prophylaxis with G-CSFs significantly reduces FN incidence in adults undergoing chemotherapy for solid tumours or lymphoma. Pegfilgrastim reduces FN incidence to a significantly greater extent than filgrastim.

\section{Background}

Neutropenia is the major dose-limiting toxicity of many chemotherapy regimens. Grade 3 and grade 4 neutropenia are defined as a neutrophil count $<1.0 \times 10^{9} / \mathrm{L}$ and $<0.5 \times 10^{9} / \mathrm{L}$ respectively. Febrile neutropenia (FN) is defined as neutropenia with fever, usually indicating infection, and is associated with substantial morbidity, mortality, and costs [1]. The direct risk of mortality associated with FN has been estimated as 9.5\% (95\% confidence interval [CI]: 9.2\%, 9.8\%) in a study of 41,779 cancer patients hospitalised with FN [1]. Management of FN often requires lengthy hospitalisation, [1] with

\footnotetext{
* Correspondence: k.l.cooper@sheffield.ac.uk

'School of Health and Related Research (SCHARR), University of Sheffield, Sheffield, UK

Full list of author information is available at the end of the article
}

associated costs and detrimental effects on quality of life $[2,3]$. In addition, an FN episode has been shown to increase the risk of chemotherapy dose reductions and delays [4]. Unplanned reductions in chemotherapy dose may cause further deaths from cancer in the long-term; in a retrospective analysis of breast cancer patients with a 30-year follow-up, the survival rate was $40 \%$ (95\% CI: $26 \%, 55 \%$ ) among patients receiving at least $85 \%$ of their planned dose, but only $21 \%$ (95\% CI: $14 \%, 26 \%$ ) among patients who received less than $85 \%$ [5].

Recombinant human granulocyte colony-stimulating factors (G-CSFs) stimulate production of mature, functional neutrophils [6]. G-CSFs have been shown to reduce the incidence of $\mathrm{FN}$ when used as prophylaxis following chemotherapy. Three G-CSFs are currently in common usage: filgrastim, pegfilgrastim, and 
lenograstim. Filgrastim and lenograstim are administered as a series of daily injections; clinical studies suggest an average of 11 injections per chemotherapy cycle are required to achieve recovery of the absolute neutrophil count (ANC) to within the normal range [7-10]. Pegfilgrastim is administered as a single injection per chemotherapy cycle $[11,12]$. G-CSFs may be administered as primary prophylaxis (in every chemotherapy cycle from cycle 1) or as secondary prophylaxis (in all remaining cycles following a neutropenic event such as FN or prolonged severe neutropenia). The overall FN risk is dependent on chemotherapy regimen as well as individual patient risk factors such as age, performance status and disease stage [13]. Guidelines from the European Organisation for Research and Treatment of Cancer (EORTC), [13] the American Society of Clinical Oncology (ASCO) [14] and the National Comprehensive Cancer Network (NCCN) [15] recommend that prophylactic G-CSFs should be used where the risk of FN associated with the chemotherapy regimen is greater than or equal to $20 \%$, and may be considered where the risk is 10 $20 \%$, particularly where additional patient risk factors are present.

This paper reports a systematic review and meta-analysis of the effect of primary G-CSF prophylaxis (with pegfilgrastim, filgrastim or lenograstim) on incidence of FN. The effect of each G-CSF is assessed in comparison with no primary G-CSF prophylaxis and in comparison with other G-CSFs.

\section{Methods}

\section{Search strategy}

The systematic review followed the recommendations in the PRISMA statement [16,17]. A systematic search was undertaken to identify randomised controlled trials (RCTs) of pegfilgrastim, filgrastim or lenograstim, compared with no primary G-CSF or with one another, for the reduction of FN following chemotherapy. A previous systematic review by Kuderer et al. [18] presented a meta-analysis of FN incidence within RCTs of primary G-CSF prophylaxis versus no primary G-CSF prophylaxis, while a systematic review by Pinto et al. [19] meta-analysed RCTs of primary prophylaxis using pegfilgrastim versus filgrastim. The literature searches within these previous reviews were conducted during 2006. Therefore, databases were searched from 2006 onwards, whereas studies published prior to 2006 were identified from the two existing reviews. Searches were undertaken in December 2009. The following databases were searched: Medline, Medline in Process, EMBASE, Science Citation Index, Cochrane Database of Systematic Reviews, Cochrane CENTRAL Register of Controlled Trials, Database of Abstracts of Reviews of Effects (DARE), Health Technology Assessment Database and
NHS Economic Evaluation Database (NHS-EED). The Medline search strategy was designed with reference to the previous two reviews, and comprised subject headings and text words for G-CSFs combined with a search filter to identify RCTs (Appendix 1). Searches were not restricted by language. Bibliographies of retrieved papers were searched for any additional relevant studies.

\section{Inclusion and exclusion criteria}

Studies were considered suitable for inclusion if they assessed primary G-CSF prophylaxis (pegfilgrastim, filgrastim or lenograstim) administered 1-3 days after the completion of chemotherapy, versus a different G-CSF or versus no primary G-CSF prophylaxis. Studies were only included if they reported incidence of FN. For consistency with the two existing systematic reviews, $[18,19]$ only studies of adult cancer patients with solid tumours or lymphoma were included. Studies allowing concomitant antibiotic prophylaxis were included if identical prophylaxis was administered in both study arms. The following study types were excluded: studies of G-CSFs for treatment of FN; studies in children; studies in patients with leukaemia, myeloid malignancies or myelodysplastic syndromes; studies of G-CSFs for stem cell mobilisation in bone marrow or peripheral blood stem cell transplantation; economic analyses; studies with differing drugs, doses or schedules of chemotherapy in each arm; studies with differing doses of the same GCSF in each arm; and studies not published in English.

\section{Outcome measures}

The outcome measure assessed in this review was the incidence of FN over all cycles of chemotherapy within each study. FN was chosen as a key clinical outcome due to its direct bearing on morbidity, mortality and hospitalisation rates, and also because this review was undertaken alongside the development of an economic model which utilised FN rate as a key parameter.

\section{Data extraction}

Data was extracted by two reviewers using a form developed for this review and any discrepancies were resolved through discussion.

\section{Data synthesis}

Meta-analyses were undertaken to compare the effectiveness of G-CSFs versus no prophylaxis and versus each other for the reduction of FN. Analyses were undertaken using RevMan software (version 5, Cochrane Collaboration). Results for each comparison were presented as a pooled relative risk and 95\% CIs. Although clinical and statistical heterogeneity existed between studies, there was insufficient data on individual populations to facilitate separate analyses. Therefore, for 
consistency with existing reviews, all studies were included in the analysis, and a random effects model was used. Heterogeneity was presented using the $\mathrm{I}^{2}$ statistic, which describes the percentage of the variability in effect estimates that is due to heterogeneity rather than sampling error (chance) [20].

\section{Results}

\section{Number and characteristics of included studies}

The flow chart for study inclusion is shown in Figure 1 and the included studies are described in Table 1. Studies published from 2006 onwards were identified from the literature search, and studies published prior to 2006 were identified from two previous reviews $[18,19]$. In total, 23 citations relating to 25 studies satisfied the inclusion criteria: 5 studies of primary pegfilgrastim vs. no primary G-CSF (within 4 citations); [21-24] 10 studies of primary filgrastim vs. no primary G-CSF (within 9 citations); [25-33] 5 studies of primary lenograstim vs. no primary G-CSF; [9,10,34-36] and 5 studies of primary pegfilgrastim vs. primary filgrastim [7,8,37-39]. No studies were identified comparing lenograstim with either pegfilgrastim alone or filgrastim alone.

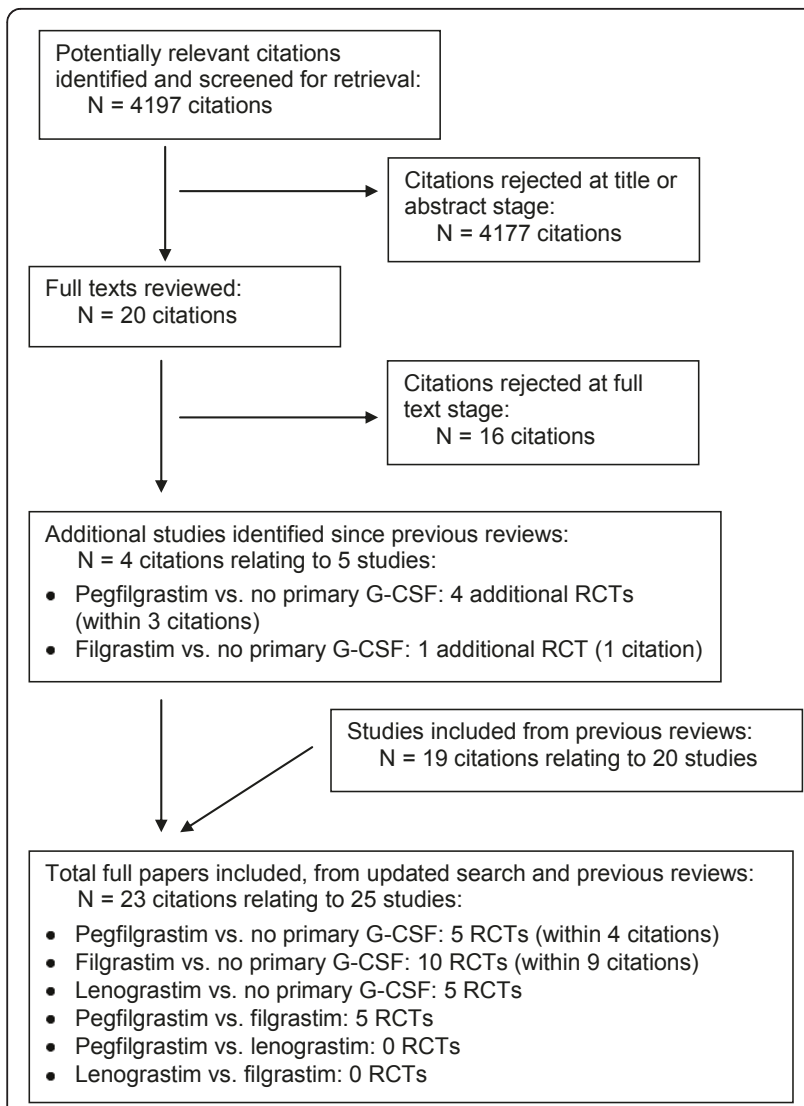

Figure 1 Flow chart for identification of relevant studies.
A previous systematic review of prophylactic G-CSF use [18] included only a single study of pegfilgrastim versus no primary G-CSF [21]. Our literature search identified 4 additional RCTs of pegfilgrastim vs. no primary G-CSF, which were conducted in populations with colorectal cancer, [24] breast cancer, [23] non-Hodgkin's lymphoma, [22] and various solid tumours; [22] the latter three studies were restricted to elderly patients. Our review also identified an additional large RCT of filgrastim vs. no primary G-CSF in breast cancer [33].

There was heterogeneity among trials of all three GCSFs in terms of cancer type, patient age, chemotherapy regimen, number of chemotherapy cycles and cycle length (Table 1). Filgrastim and lenograstim were generally given for 10-14 days where the chemotherapy cycle length was 3 weeks (and for fewer days in a small number of trials with shorter cycle lengths). The comparator arm in some of the studies included secondary G-CSFs for those patients having an FN event, and some trials allowed prophylactic antibiotics in both arms. Some studies were open-label rather than double-blind.

\section{Effectiveness of G-CSFs in reducing febrile neutropenia incidence}

The relative risks of FN incidence are shown in Figure 2 for trials of G-CSF versus no primary G-CSF, and in Figure 3 for trials of pegfilgrastim versus filgrastim. The pooled relative risks for each G-CSF comparison are summarised in Table 2. Primary prophylaxis with each of the G-CSFs significantly decreased the risk of FN compared with no primary G-CSF, with relative risks of 0.30 (95\% CI: 0.14 to 0.65 ) for pegfilgrastim, 0.57 (95\% CI: 0.48 to 0.69 ) for filgrastim, and 0.62 (95\% CI: 0.44 to 0.88) for lenograstim. Overall, the relative risk of FN when using any primary G-CSF prophylaxis versus no primary G-CSF prophylaxis was 0.51 (95\% CI: 0.41 to $0.62)$.

There was a relatively high level of statistical heterogeneity in the analyses as shown by the $\mathrm{I}^{2}$ statistic, which ranged from $50-76 \%$; this is likely to reflect the variations between studies in factors such as cancer type, patient age, chemotherapy regimen, number of chemotherapy cycles and cycle length. Individual studies differed on too many variables for formal sub-analyses to be meaningful. However, Figures 2 and 3 illustrate the cancer type for each study (shown after the author and date) and highlight studies in populations aged $\geq 60$ or $\geq 65$ years (shown by asterisks). There was no clear difference in G-CSF effectiveness between cancer types, nor in studies restricting to an elderly population. As the majority of studies administered filgrastim and lenograstim for 10-14 days (for 3-week chemotherapy cycles), there was insufficient data to assess the effects of shorter durations of G-CSF treatment. 
Table 1 Description of trials of primary G-CSFs (vs. no primary G-CSF, or vs. each other)

\begin{tabular}{|c|c|c|c|c|c|c|c|c|c|c|c|c|c|c|}
\hline Trial & $\begin{array}{l}\text { Study } \\
\text { design }\end{array}$ & Cancer type & $\begin{array}{l}\text { Cancer } \\
\text { stage }\end{array}$ & $\begin{array}{l}\text { Patient } \\
\text { age }\end{array}$ & $\begin{array}{l}\text { Chemotherapy } \\
\text { regimen }\end{array}$ & $\begin{array}{l}N \text { cycles } \\
\text { (max) }\end{array}$ & $\begin{array}{l}\text { Cycle } \\
\text { length }\end{array}$ & $\begin{array}{l}\text { Arm } 1 \mathrm{G}-\mathrm{CSF} \\
\text { strategy }^{\mathrm{b}}\end{array}$ & $\begin{array}{l}\text { Arm 1: } \\
\mathrm{N} \\
\text { analysed }\end{array}$ & $\begin{array}{l}\text { Arm 1: } \\
\text { days } \\
\text { primary } \\
\text { G-CSF }\end{array}$ & $\begin{array}{l}\text { Arm } 2 \text { G-CSF } \\
\text { strategy }^{b}\end{array}$ & $\begin{array}{l}\text { Arm 2: } \\
\mathrm{N} \\
\text { analysed }\end{array}$ & $\begin{array}{l}\text { Arm 2: } \\
\text { days } \\
\text { primary } \\
\text { G-CSF }\end{array}$ & $\begin{array}{l}\text { FN } \\
\text { definition }\end{array}$ \\
\hline \multicolumn{15}{|l|}{$\begin{array}{l}\text { Pegfilgrastim } \\
\text { vs. no } \\
\text { primary } \\
\text { G-CSF }\end{array}$} \\
\hline $\begin{array}{l}\text { a Romieu } 2007 \\
\text { [23] }\end{array}$ & $\begin{array}{l}\text { RCT, } \\
\text { phase } \\
\text { II, OL }\end{array}$ & $\begin{array}{l}\text { Breast } \\
\text { cancer }\end{array}$ & $\begin{array}{l}\text { Stage }\|-I\|, \\
\text { node- } \\
\text { positive }\end{array}$ & $\begin{array}{l}\text { Age } \geq \\
65 . \\
\text { Median } \\
68, \\
\text { range } \\
65-77\end{array}$ & FEC-100 & $\begin{array}{l}6 \text { (FN } \\
\text { reported } \\
\text { cycle } 1 \\
\text { only) }\end{array}$ & 3 weeks & $\begin{array}{l}\text { Pegfilgrastim } \\
\text { primary: } 6 \text { mg day } 2\end{array}$ & 30 & 1 & $\begin{array}{l}\text { No primary G- } \\
\text { CSF, } \\
\text { pegfilgrastim } \\
\text { secondary } \\
\text { (following FN } \\
\text { or } \\
\text { neutropenia) }\end{array}$ & 29 & 0 & $\begin{array}{l}\text { Fever }+ \\
\text { ANC }<1 \\
\times 10^{9} / 1\end{array}$ \\
\hline Vogel 2005[21] & $\begin{array}{l}\text { RCT, } \\
\text { phase } \\
\text { III, DB }\end{array}$ & $\begin{array}{l}\text { Breast } \\
\text { cancer }\end{array}$ & $\begin{array}{l}62 \% \text { stage } \\
\text { IV, 38\% } \\
\text { other } \\
\text { stages }\end{array}$ & $\begin{array}{l}\text { Mean } \\
\text { age 52, } \\
\text { range } \\
21-88\end{array}$ & $\begin{array}{l}\text { Docetaxel } 100 \\
\mathrm{mg} / \mathrm{m}^{2}\end{array}$ & 4 & 3 weeks & $\begin{array}{l}\text { Pegfilgrastim } \\
\text { primary: } 6 \text { mg day } 2\end{array}$ & 463 & 1 & $\begin{array}{l}\text { Placebo } \\
\text { primary, } \\
\text { pegfilgrastim } \\
\text { secondary } \\
\text { (following FN) }\end{array}$ & 465 & 0 & $\begin{array}{l}\text { Fever }+ \\
\text { ANC }<0.5 \\
\times 10^{9} / 1\end{array}$ \\
\hline $\begin{array}{l}\text { a Hecht } 2009 \\
\text { [24] }\end{array}$ & $\begin{array}{l}\text { RCT, } \\
\text { phase } \\
\|\end{array}$ & $\begin{array}{l}\text { Colorectal } \\
\text { cancer }\end{array}$ & NR & NR & $\begin{array}{l}\text { FOLFOX (49\%), } \\
\text { FOLFIRI (26\%) or } \\
\text { FOIL (25\%) }\end{array}$ & 4 & 2 weeks & $\begin{array}{l}\text { Pegfilgrastim } \\
\text { primary: } 6 \text { mg day } 4\end{array}$ & 123 & 1 & $\begin{array}{l}\text { Placebo } \\
\text { primary }\end{array}$ & 118 & 0 & $\begin{array}{l}\text { Grade 3-4 } \\
\text { FN } \\
\text { (assumed } \\
\text { fever }+ \\
\text { ANC }<1 \\
\times 10^{9} / \text { I) }\end{array}$ \\
\hline $\begin{array}{l}\text { Balducci } \\
\text { 2007: NHL[22] }\end{array}$ & $\begin{array}{l}\mathrm{RCT}, \\
\mathrm{OL}\end{array}$ & $\mathrm{NHL}$ & $\begin{array}{l}38 \% \text { stage } \\
\text { I-II, } 62 \% \\
\text { stage III-IV }\end{array}$ & $\begin{array}{l}\text { Age } \geq \\
65 . \\
\text { Median } \\
72, \\
\text { range } \\
65-88\end{array}$ & $\begin{array}{l}\text { CHOP or R- } \\
\text { CHOP }\end{array}$ & 6 & 3 weeks & $\begin{array}{l}\text { Pegfilgrastim } \\
\text { primary: } 6 \mathrm{mg} \text { day } 2\end{array}$ & 73 & 1 & $\begin{array}{l}\text { No primary G- } \\
\text { CSF, } \\
\text { pegfilgrastim } \\
\text { secondary (at } \\
\text { physician's } \\
\text { discretion) }\end{array}$ & 73 & 0 & $\begin{array}{l}\text { Fever }+ \\
\text { ANC }<1 \\
\times 10^{9} / 1\end{array}$ \\
\hline $\begin{array}{l}\text { Balducci } \\
\text { 2007: solid } \\
\text { tumour[22] }\end{array}$ & $\begin{array}{l}\mathrm{RCT} \\
\mathrm{OL}\end{array}$ & $\begin{array}{l}\text { Solid tumour } \\
\text { (lung, } \\
\text { ovarian, } \\
\text { breast) }\end{array}$ & $\begin{array}{l}\text { 31\% stage } \\
\text { I-II, } 69 \% \\
\text { stage III-IV }\end{array}$ & $\begin{array}{l}\text { Age } \geq \\
65 . \\
\text { Median } \\
72, \\
\text { range } \\
65-88\end{array}$ & $\begin{array}{l}\text { One of } 15 \\
\text { regimens with } \\
\text { mild-to- } \\
\text { moderate risk of } \\
\text { neutropenia }\end{array}$ & 6 & 3 weeks & $\begin{array}{l}\text { Pegfilgrastim } \\
\text { primary: } 6 \mathrm{mg} \text { day } 2\end{array}$ & 343 & 1 & $\begin{array}{l}\text { No primary G- } \\
\text { CSF, } \\
\text { pegfilgrastim } \\
\text { secondary (at } \\
\text { physician's } \\
\text { discretion) }\end{array}$ & 343 & 0 & $\begin{array}{l}\text { Fever }+ \\
\text { ANC }<1 \\
\times 10^{9} / 1\end{array}$ \\
\hline \multicolumn{15}{|l|}{$\begin{array}{l}\text { Filgrastim vs. } \\
\text { no primary } \\
\text { G-CSF }\end{array}$} \\
\hline $\begin{array}{l}\text { a del Giglio } \\
2008[33]\end{array}$ & $\begin{array}{l}\mathrm{RCT} \\
\mathrm{DB}\end{array}$ & $\begin{array}{l}\text { Breast } \\
\text { cancer }\end{array}$ & $\begin{array}{l}21 \% \text { high- } \\
\text { risk stage } \\
{ }_{1}, \\
53 \% \text { stage } \\
\text { III, } \\
25 \% \text { stage } \\
\text { IV }\end{array}$ & $\begin{array}{l}\text { Mean } \\
\text { age 51, } \\
\text { range } \\
25-75\end{array}$ & $\begin{array}{l}\text { Doxorubicin } 60 \\
\mathrm{mg} / \mathrm{m}^{2} / \\
\text { docetaxel } 75 \\
\mathrm{mg} / \mathrm{m}^{2}\end{array}$ & $\begin{array}{l}4 \text { (FN } \\
\text { reported } \\
\text { cycle } 1 \\
\text { only) }\end{array}$ & 3 weeks & $\begin{array}{l}\text { Filgrastim primary } \\
\text { (Neupogen or } \\
\text { XM02): } 5 \mathrm{ug} / \mathrm{kg} / \mathrm{d} \\
\text { from day } 2 \mathrm{up} \text { to } \\
14 \mathrm{~d} \text { or to ANC }=10 \\
\times 10^{9} / \mathrm{l}\end{array}$ & 276 & $\begin{array}{l}5 \text { to } 14 \\
\text { (median } \\
9-10 \text { ) }\end{array}$ & $\begin{array}{l}\text { Placebo in } \\
\text { cycle 1; } \\
\text { filgrastim } \\
\text { (XM02) in } \\
\text { subsequent } \\
\text { cycles }\end{array}$ & 72 & $\begin{array}{l}0 \text { (cycle } \\
\text { 1) }\end{array}$ & $\begin{array}{l}\text { Fever }+ \\
\text { ANC }<0.5 \\
\times 10^{9} / 1\end{array}$ \\
\hline
\end{tabular}


Table 1 Description of trials of primary G-CSFs (vs. no primary G-CSF, or vs. each other) (Continued)

\begin{tabular}{|c|c|c|c|c|c|c|c|c|c|c|c|c|c|c|}
\hline $\begin{array}{l}\text { Timmer-Bonte } \\
\text { 2005[29] }\end{array}$ & $\begin{array}{l}\text { RCT, } \\
\text { phase } \\
\text { III, OL }\end{array}$ & SCLC & $\begin{array}{l}69 \% \\
\text { extensive, } \\
31 \% \\
\text { limited }\end{array}$ & $\begin{array}{l}\text { Age } \\
\text { range } \\
36-81\end{array}$ & $\mathrm{CDE}$ & 5 & 3 weeks & $\begin{array}{l}\text { Filgrastim primary: } \\
300 / 450 \text { ug/d from } \\
\text { day } 4 ; \text { prophylactic } \\
\text { antibiotics }\end{array}$ & 90 & 10 & $\begin{array}{l}\text { No primary G- } \\
\text { CSF; } \\
\text { prophylactic } \\
\text { antibiotics }\end{array}$ & 85 & 0 & $\begin{array}{l}\text { Fever }+ \\
\text { ANC }<0.5 \\
\times 10^{9} / 1\end{array}$ \\
\hline $\begin{array}{l}\text { Trillet-Lenoir } \\
\text { 1993[30] }\end{array}$ & $\begin{array}{l}\mathrm{RCT}, \\
\mathrm{DB}\end{array}$ & SCLC & $\begin{array}{l}64 \% \\
\text { extensive, } \\
36 \% \\
\text { limited }\end{array}$ & $\begin{array}{l}\text { Median } \\
59\end{array}$ & CDE & 6 & 3 weeks & $\begin{array}{l}\text { Filgrastim primary: } \\
230 \mathrm{ug} / \mathrm{m}^{2} / \mathrm{d} \text { from } \\
\text { day } 4 \mathrm{up} \text { to } 14 \mathrm{~d} \text { or } \\
\text { until ANC }=10 \times \\
10^{9} / \mathrm{l}\end{array}$ & 65 & 9 to 14 & $\begin{array}{l}\text { Placebo } \\
\text { primary }\end{array}$ & 64 & 0 & $\begin{array}{l}\text { Fever }+ \\
\text { ANC }<1 \\
\times 10^{9} / 1\end{array}$ \\
\hline $\begin{array}{l}\text { Crawford } 1991 \\
\text { [31] }\end{array}$ & $\begin{array}{l}\mathrm{RCT} \\
\mathrm{DB}\end{array}$ & SCLC & $\begin{array}{l}72 \% \\
\text { extensive, } \\
28 \% \\
\text { limited }\end{array}$ & $\begin{array}{l}\text { Age } \\
\text { range } \\
31-80\end{array}$ & $\mathrm{CDE}$ & 6 & 3 weeks & $\begin{array}{l}\text { Filgrastim primary: } \\
230 \mathrm{ug} / \mathrm{m}^{2} / \mathrm{d} \text { from } \\
\text { day } 4 \mathrm{up} \text { to } 14 \mathrm{~d} \text { or } \\
\text { until ANC }=10 \times \\
10^{9} / \mathrm{l}\end{array}$ & 95 & 9 to 14 & $\begin{array}{l}\text { Placebo } \\
\text { primary; } \\
\text { secondary G- } \\
\text { CSF }\end{array}$ & 104 & 0 & $\begin{array}{l}\text { Fever }+ \\
\text { ANC }<1 \\
\times 10^{9} / 1\end{array}$ \\
\hline $\begin{array}{l}\text { Doorduijn } \\
\text { 2003[25] }\end{array}$ & $\begin{array}{l}\text { RCT, } \\
\mathrm{OL}\end{array}$ & $\begin{array}{l}\text { Aggressive } \\
\mathrm{NHL}\end{array}$ & Stage II-IV & $\begin{array}{l}\text { Age } \geq \\
65 . \\
\text { Median } \\
72 \\
\text { range } \\
65-90\end{array}$ & $\mathrm{CHOP}$ & 6 to 8 & 3 weeks & $\begin{array}{l}\text { Filgrastim primary: } \\
300 \mathrm{ug} / \mathrm{d} \text { from day } 2 \\
\text { for } 10 \mathrm{~d}\end{array}$ & 197 & 10 & $\begin{array}{l}\text { No primary G- } \\
\text { CSF }\end{array}$ & 192 & 0 & $\begin{array}{l}\text { FN not } \\
\text { defined in } \\
\text { terms of } \\
\text { ANC }\end{array}$ \\
\hline $\begin{array}{l}\text { Osby } 2003 \\
\text { (CHOP)[26] }\end{array}$ & $\begin{array}{l}\text { RCT, } \\
\mathrm{OL}\end{array}$ & $\begin{array}{l}\text { Aggressive } \\
\mathrm{NHL}\end{array}$ & Stage II-IV & $\begin{array}{l}\text { Age } \geq \\
60 \text {. } \\
\text { Median } \\
71, \\
\text { range } \\
60-86\end{array}$ & $\mathrm{CHOP}$ & $\begin{array}{l}4 \text { to } 8 \\
\text { (most } 8 \text { ) }\end{array}$ & 3 weeks & $\begin{array}{l}\text { Filgrastim primary: } 5 \\
\text { ug/kg/d from day } 2 \\
\text { up to } 14 \mathrm{~d} \text { or until } \\
\text { ANC }=10 \times 10^{9} / \mathrm{l}\end{array}$ & 101 & 10 to 14 & $\begin{array}{l}\text { No primary G- } \\
\text { CSF }\end{array}$ & 104 & 0 & $\begin{array}{l}\text { Fever }+ \\
\text { ANC }<0.5 \\
\times 10^{9} / 1\end{array}$ \\
\hline $\begin{array}{l}\text { Osby } 2003 \\
\text { (CNOP)[26] }\end{array}$ & $\begin{array}{l}\mathrm{RCT}, \\
\mathrm{OL}\end{array}$ & $\begin{array}{l}\text { Aggressive } \\
\mathrm{NHL}\end{array}$ & Stage II-IV & $\begin{array}{l}\text { Age } \geq \\
60 \text {. } \\
\text { Median } \\
71 \\
\text { range } \\
60-86\end{array}$ & CNOP & $\begin{array}{l}4 \text { to } 8 \\
\text { (most } 8 \text { ) }\end{array}$ & 3 weeks & $\begin{array}{l}\text { Filgrastim primary: } 5 \\
\text { ug/kg/d from day } 2 \\
\text { up to } 14 \mathrm{~d} \text { or until } \\
\text { ANC }=10 \times 10^{9} / \mathrm{l}\end{array}$ & 125 & 10 to 14 & $\begin{array}{l}\text { No primary G- } \\
\text { CSF }\end{array}$ & 125 & 0 & $\begin{array}{l}\text { Fever }+ \\
\text { ANC }<0.5 \\
\times 10^{9} / 1\end{array}$ \\
\hline $\begin{array}{l}\text { Zinzani } 1997 \\
\text { [27] }\end{array}$ & $\begin{array}{l}\text { RCT, } \\
\mathrm{OL}\end{array}$ & $\begin{array}{l}\text { Aggressive } \\
\mathrm{NHL}\end{array}$ & Stage II-IV & $\begin{array}{l}\text { Age } \geq \\
60 . \text { Age } \\
\text { range } \\
60-82\end{array}$ & VNCOP-B & 8 & $\begin{array}{l}1 \text { week } \\
\text { (differs } \\
\text { alternate } \\
\text { weeks) }\end{array}$ & $\begin{array}{l}\text { Filgrastim primary: } 5 \\
\text { ug/kg/d from day } 3 ; \\
\text { prophylactic } \\
\text { antibiotics }\end{array}$ & 77 & 5 & $\begin{array}{l}\text { No primary G- } \\
\text { CSF; } \\
\text { prophylactic } \\
\text { antibiotics }\end{array}$ & 72 & 0 & $\begin{array}{l}\text { FN not } \\
\text { defined in } \\
\text { terms of } \\
\text { ANC }\end{array}$ \\
\hline $\begin{array}{l}\text { Pettengell } \\
1992[28]\end{array}$ & $\begin{array}{l}\text { RCT, } \\
\mathrm{OL}\end{array}$ & $\begin{array}{l}\text { Aggressive } \\
\mathrm{NHL}\end{array}$ & Any stage & $\begin{array}{l}\text { Age } \\
\text { range } \\
16-71\end{array}$ & VAPEC-B & 11 & $\begin{array}{l}1 \text { week } \\
\text { (differs } \\
\text { alternate } \\
\text { weeks) }\end{array}$ & $\begin{array}{l}\text { Filgrastim primary: } \\
230 \mathrm{ug} / \mathrm{m}^{2} / \mathrm{d} \text { from } \\
\text { day } 2 \mathrm{up} \text { to } 14 \mathrm{~d} \text { or } \\
\text { until ANC }=10 \times \\
10^{9} / \text {; prophylactic } \\
\text { antibiotics }\end{array}$ & 41 & 12 & $\begin{array}{l}\text { No primary G- } \\
\text { CSF; } \\
\text { prophylactic } \\
\text { antibiotics }\end{array}$ & 39 & 0 & $\begin{array}{l}\text { Fever }+ \\
\text { ANC }<1 \\
\times 10^{9} / 1\end{array}$ \\
\hline Fossa 1998[32] & $\begin{array}{l}\text { RCT, } \\
\text { phase } \\
\text { III, OL }\end{array}$ & $\begin{array}{l}\text { Germ cell } \\
\text { cancer }\end{array}$ & $\begin{array}{l}\text { Metastatic, } \\
\text { poor- } \\
\text { prognosis }\end{array}$ & $\begin{array}{l}\text { Age } \\
\text { range } \\
15-65\end{array}$ & $\begin{array}{l}\text { BEP/EP or BOP/ } \\
\text { VIP-B }\end{array}$ & 6 & $\begin{array}{l}3 \text { weeks } \\
\text { or } 10 \mathrm{~d}\end{array}$ & $\begin{array}{l}\text { Filgrastim primary: } 5 \\
\text { ug/kg/d from day } 3 \\
\text { or } 6\end{array}$ & 129 & 7 or 14 & $\begin{array}{l}\text { No primary G- } \\
\text { CSF }\end{array}$ & 130 & 0 & $\begin{array}{l}\text { FN not } \\
\text { defined in } \\
\text { terms of } \\
\text { ANC }\end{array}$ \\
\hline
\end{tabular}


Table 1 Description of trials of primary G-CSFs (vs. no primary G-CSF, or vs. each other) (Continued)

\begin{tabular}{|c|c|c|c|c|c|c|c|c|c|c|c|c|c|c|}
\hline $\begin{array}{l}\text { Lenograstim } \\
\text { vs. no } \\
\text { primary G- } \\
\text { CSF }\end{array}$ & & & & & & & & & & & & & & \\
\hline $\begin{array}{l}\text { Chevallier } \\
\text { 1995[9] }\end{array}$ & $\begin{array}{l}\mathrm{RCT} \\
\mathrm{DB}\end{array}$ & $\begin{array}{l}\text { Breast } \\
\text { cancer, } \\
\text { inflammatory }\end{array}$ & $\begin{array}{l}\text { Non- } \\
\text { metastatic }\end{array}$ & $\begin{array}{l}\text { Age } \\
\text { range } \\
23-65\end{array}$ & FEC-high-dose & 4 & 3 weeks & $\begin{array}{l}\text { Lenograstim primary: } \\
5 \mathrm{ug} / \mathrm{kg} / \mathrm{d} \text { from day } \\
6\end{array}$ & 61 & 10 & $\begin{array}{l}\text { Placebo } \\
\text { primary }\end{array}$ & 59 & 0 & $\begin{array}{l}\text { Fever }+ \\
\text { ANC }<1 \\
\times 10^{9} / 1 \\
\end{array}$ \\
\hline $\begin{array}{l}\text { Gisselbrecht } \\
\text { 1997[34] }\end{array}$ & $\begin{array}{l}\mathrm{RCT} \\
\mathrm{DB}\end{array}$ & $\begin{array}{l}\text { Aggressive } \\
\mathrm{NHL}\end{array}$ & Any stage & $\begin{array}{l}\text { Age } \\
\text { range } \\
15-55\end{array}$ & $\begin{array}{l}\text { LNH-87 (LNH-84 } \\
\text { + randomization } \\
\text { to } \\
\text { anthracyclines) }\end{array}$ & 4 & 2 weeks & $\begin{array}{l}\text { Lenograstim primary: } \\
5 \mathrm{ug} / \mathrm{kg} / \mathrm{d} \text { from day } \\
6\end{array}$ & 82 & 8 & $\begin{array}{l}\text { Placebo } \\
\text { primary }\end{array}$ & 80 & 0 & $\begin{array}{l}\text { Fever }+ \\
\text { ANC }<1 \\
\times 10^{9} / l\end{array}$ \\
\hline Bui 1995[10] & $\begin{array}{l}\mathrm{RCT} \\
\mathrm{DB}\end{array}$ & $\begin{array}{l}\text { Soft tissue } \\
\text { sarcoma }\end{array}$ & $\begin{array}{l}\text { Metastatic } \\
\text { or locally } \\
\text { advanced }\end{array}$ & $\begin{array}{l}\text { Age } \\
\text { range } \\
21-69\end{array}$ & MAID & $\begin{array}{l}6(\mathrm{FN} \\
\text { reported } \\
\text { cycle } 1 \\
\text { only) }\end{array}$ & 3 weeks & $\begin{array}{l}\text { Lenograstim primary: } \\
5 \mathrm{ug} / \mathrm{kg} / \mathrm{d} \text { from day } \\
4 \mathrm{up} \text { to } 14 \mathrm{~d} \text { or until } \\
\text { ANC }=30 \times 10^{9} / \mathrm{l}\end{array}$ & 22 & 10 to 14 & $\begin{array}{l}\text { Placebo } \\
\text { primary; } \\
\text { secondary G- } \\
\text { CSF }\end{array}$ & 26 & 0 & $\begin{array}{l}\text { Fever }+ \\
\text { ANC }<1 \\
\times 10^{9} / 1\end{array}$ \\
\hline $\begin{array}{l}\text { Gebbia } 1994 \\
\text { [35] }\end{array}$ & $\begin{array}{l}\mathrm{RCT} \\
\mathrm{DB}\end{array}$ & Various & Advanced & $\begin{array}{l}\text { Age } \\
\text { range } \\
40-75\end{array}$ & Various & Various & Various & $\begin{array}{l}\text { Lenograstim primary: } \\
5 \mathrm{ug} / \mathrm{kg} / \mathrm{d}\end{array}$ & 23 & $\geq 7 d$ & $\begin{array}{l}\text { Placebo } \\
\text { primary }\end{array}$ & 28 & 0 & $\begin{array}{l}\text { Fever }+ \\
\text { ANC }<1 \\
\times 10^{9} / 1\end{array}$ \\
\hline $\begin{array}{l}\text { Gebbia } 1993 \\
\text { [36] }\end{array}$ & $\begin{array}{l}\mathrm{RCT} \\
\mathrm{DB}\end{array}$ & Various & Advanced & $\begin{array}{l}\text { Age } \\
\text { range } \\
38-66\end{array}$ & Various & Various & Various & $\begin{array}{l}\text { Lenograstim primary: } \\
5 \mathrm{ug} / \mathrm{kg} / \mathrm{d}\end{array}$ & 43 & 7 to 10 & $\begin{array}{l}\text { Placebo } \\
\text { primary }\end{array}$ & 43 & 0 & $\begin{array}{l}\text { Fever }+ \\
\text { ANC }<1 \\
\times 10^{9} / 1 \\
\end{array}$ \\
\hline \multicolumn{15}{|l|}{$\begin{array}{l}\text { Pegfilgrastim } \\
\text { vs. } 10 \text { - or } 11- \\
\text { day filgrastim }\end{array}$} \\
\hline Green 2003[7] & $\begin{array}{l}\text { RCT, } \\
\text { phase } \\
\text { III, DB }\end{array}$ & $\begin{array}{l}\text { Breast } \\
\text { cancer }\end{array}$ & $\begin{array}{l}28 \% \text { stage } \\
\text { II, } 27 \% \\
\text { stage III, } \\
45 \% \text { stage } \\
\text { IV }\end{array}$ & $\begin{array}{l}\text { Mean } \\
\text { age } 52, \\
\text { range } \\
30-75\end{array}$ & $\begin{array}{l}\text { Doxorubicin } 60 \\
\mathrm{mg} / \mathrm{m}^{2} / \\
\text { docetaxel } 75 \\
\mathrm{mg} / \mathrm{m}^{2}\end{array}$ & 4 & 3 weeks & $\begin{array}{l}\text { Pegfilgrastim } \\
\text { primary: } 6 \text { mg day 2; } \\
\text { then placebo up to } \\
14 d\end{array}$ & 77 & 1 & $\begin{array}{l}\text { Filgrastim } \\
\text { primary: } 5 \text { ug/ } \\
\text { kg, from day } 2 \\
\text { up to } 14 \mathrm{~d} \text { or } \\
\text { until ANC }=10 \\
\times 10^{9} / \mathrm{l}\end{array}$ & 75 & $\begin{array}{l}11 \\
\text { (median) }\end{array}$ & $\begin{array}{l}\text { Fever }+ \\
\text { ANC }<0.5 \\
\times 10^{9} / \mathrm{I}\end{array}$ \\
\hline $\begin{array}{l}\text { Holmes 2002: } \\
\text { phase III[8] }\end{array}$ & $\begin{array}{l}\text { RCT, } \\
\text { phase } \\
\text { III, DB }\end{array}$ & $\begin{array}{l}\text { Breast } \\
\text { cancer }\end{array}$ & $\begin{array}{l}\text { High-risk } \\
\text { stage II, III } \\
\text { or IV. 37\% } \\
\text { stage IV }\end{array}$ & $\begin{array}{l}\text { Mean } \\
\text { age } 51\end{array}$ & $\begin{array}{l}\text { Doxorubicin } 60 \\
\mathrm{mg} / \mathrm{m}^{2} / \\
\text { docetaxel } 75 \\
\mathrm{mg} / \mathrm{m}^{2}\end{array}$ & 4 & 3 weeks & $\begin{array}{l}\text { Pegfilgrastim } \\
\text { primary: } 100 \mathrm{ug} / \mathrm{kg} \\
\text { day } 2 ; \text { then placebo } \\
\text { up to } 14 \mathrm{~d}\end{array}$ & 149 & 1 & $\begin{array}{l}\text { Filgrastim } \\
\text { primary: } 5 \text { ug/ } \\
\text { kg, from day } 2 \\
\text { up to } 14 \mathrm{~d} \text { or } \\
\text { until ANC }=10 \\
\times 10^{9} / \mathrm{l}\end{array}$ & 147 & $\begin{array}{l}11 \\
\text { (mean) }\end{array}$ & $\begin{array}{l}\text { Fever }+ \\
\text { ANC }<0.5 \\
\times 10^{9} / l\end{array}$ \\
\hline $\begin{array}{l}\text { Holmes 2002: } \\
\text { phase II[37] }\end{array}$ & $\begin{array}{l}\mathrm{RCT} \\
\text { phase } \\
\text { II, DF }\end{array}$ & $\begin{array}{l}\text { Breast } \\
\text { cancer }\end{array}$ & $\begin{array}{l}\text { High-risk } \\
\text { stage II, III } \\
\text { or IV. 30\% } \\
\text { stage IV }\end{array}$ & $\begin{array}{l}\text { Mean } \\
\text { age } 49\end{array}$ & $\begin{array}{l}\text { Doxorubicin } 60 \\
\mathrm{mg} / \mathrm{m}^{2} / \\
\text { docetaxel } 75 \\
\mathrm{mg} / \mathrm{m}^{2}\end{array}$ & 4 & 3 weeks & $\begin{array}{l}\text { Pegfilgrastim } \\
\text { primary: } 100 \mathrm{ug} / \mathrm{kg} \\
\text { day } 2 \text { (other dose } \\
\text { groups not included } \\
\text { here) }\end{array}$ & 46 & 1 & $\begin{array}{l}\text { Filgrastim } \\
\text { primary: } 5 \text { ug/ } \\
\text { kg, from day } 2 \\
\text { up to } 14 \mathrm{~d} \text { or } \\
\text { until ANC }=10 \\
\times 10^{9} / \mathrm{l}\end{array}$ & 25 & $\begin{array}{l}10.6 ; \\
10.2 ; \\
10.4 ; \\
11.0 \\
\text { (mean } \\
\text { in cycles } \\
1-4 \text { ) }\end{array}$ & $\begin{array}{l}\text { Fever }+ \\
\text { ANC }<0.5 \\
\times 10^{9} / /\end{array}$ \\
\hline
\end{tabular}


Table 1 Description of trials of primary G-CSFs (vs. no primary G-CSF, or vs. each other) (Continued)

\begin{tabular}{|c|c|c|c|c|c|c|c|c|c|c|c|c|c|c|}
\hline Grigg 2003[38] & $\begin{array}{l}\text { RCT, } \\
\text { phase } \\
\text { II, OL, } \\
\text { DF }\end{array}$ & $\mathrm{NHL}$ & Any stage & $\begin{array}{l}\text { Age } \geq \\
60 . \\
\text { Mean } \\
68, \\
\text { range } \\
60-82\end{array}$ & $\mathrm{CHOP}$ & 6 & 3 weeks & $\begin{array}{l}\text { Pegfilgrastim } \\
\text { primary: } 100 \mathrm{ug} / \mathrm{kg} \\
\text { day } 2 \text { (other dose } \\
\text { groups not included } \\
\text { here) }\end{array}$ & 14 & 1 & $\begin{array}{l}\text { Filgrastim } \\
\text { primary: } 5 \mathrm{ug} / \\
\text { kg, from day } 2 \\
\text { up to } 14 \mathrm{~d} \text { or } \\
\text { until ANC }=10 \\
\times 10^{9} / \mathrm{l}\end{array}$ & 13 & $\begin{array}{l}10 \\
\text { (mean) }\end{array}$ & $\begin{array}{l}\text { Fever }+ \\
\text { ANC }<0.5 \\
\times 10^{9} / 1\end{array}$ \\
\hline Vose 2003[39] & $\begin{array}{l}\mathrm{RCT} \\
\text { phase } \\
\mathrm{Il}, \mathrm{OL}\end{array}$ & $\begin{array}{l}\text { NHL }(n=56) \\
\text { or } H L(n= \\
\text { 4) }\end{array}$ & $\begin{array}{l}\text { Relapsed } \\
\text { or } \\
\text { refractory }\end{array}$ & $\begin{array}{l}\text { Mean } \\
\text { age } 49 . \\
85 \%< \\
65\end{array}$ & ESHAP & $\begin{array}{l}4 \text { (FN } \\
\text { reported } \\
\text { cycles } 1 \\
\& 2 \text { only) }\end{array}$ & 3 weeks & $\begin{array}{l}\text { Pegfilgrastim } \\
\text { primary: } 100 \mathrm{ug} / \mathrm{kg} \\
\text { day } 2\end{array}$ & 29 & 1 & $\begin{array}{l}\text { Filgrastim } \\
\text { primary: } 5 \mathrm{ug} / \\
\text { kg, from day } 2 \\
\text { up to } 12 \mathrm{~d} \text { or } \\
\text { until } \mathrm{ANC}=10 \\
\times 10^{9} /\end{array}$ & 31 & $\begin{array}{l}11 \\
\text { (median) }\end{array}$ & $\begin{array}{l}\text { Fever }+ \\
\text { ANC }<0.5 \\
\times 10^{9} / 1\end{array}$ \\
\hline
\end{tabular}

a Studies added as a result of updated search. ${ }^{\mathrm{b}} \mathrm{G}$-CSF strategy: Primary prophylaxis is in all cycles. Secondary prophylaxis is in all cycles following $\mathrm{FN}$, or following $\mathrm{FN}$ or neutropenia, or at physician's discretion (as noted for individual studies). ANC = absolute neutrophil count; $\mathrm{DB}=$ double-blind; $\mathrm{DF}=$ dose-finding; $\mathrm{HL}=$ Hodgkin's lymphoma; $\mathrm{NHL}=$ non-Hodgkin's lymphoma; $\mathrm{OL}=\mathrm{open}-\mathrm{label} ; \mathrm{SCLC}=\mathrm{small}-\mathrm{cell}$ lung cancer. Chemotherapy regimens used: BEP/EP = etoposide $100 \mathrm{mg} / \mathrm{m}^{2}$, cisplatin $20 \mathrm{mg} / \mathrm{m}^{2}$, plus or minus bleomycin $30 \mathrm{U}$. BOP/NIP-B = bleomycin $30 \mathrm{U}$, vincristine $2 \mathrm{mg}$, cisplatin $20-50 \mathrm{mg} / \mathrm{m}^{2} /$ etoposide $100 \mathrm{mg} / \mathrm{m}^{2}$,

ifosfamide $1000 \mathrm{mg} / \mathrm{m}^{2}$. CDE = cyclophosphamide $1 \mathrm{~g} / \mathrm{m}^{2}$, doxorubicin $45-50 \mathrm{mg} / \mathrm{m}^{2}$, etoposide $100-120 \mathrm{mg} / \mathrm{m}^{2}$. CHOP = cyclophosphamide $750 \mathrm{mg} / \mathrm{m}^{2}$, doxorubicin $50 \mathrm{mg} / \mathrm{m}^{2}$, vincristine $1.4 \mathrm{mg} / \mathrm{m}^{2}$, prednisolone $100 \mathrm{mg}$ days $1-5 . \mathrm{CNOP}=$ cyclophosphamide $750 \mathrm{mg} / \mathrm{m}^{2}$, mitoxantrone $10 \mathrm{mg} / \mathrm{m}^{2}$, vincristine $1.4 \mathrm{mg} / \mathrm{m}^{2}$, prednisolone $50 \mathrm{mg} / \mathrm{m}^{2}$ days $1-5$. ESHAP = etoposide $40 \mathrm{mg} / \mathrm{m}^{2}, \mathrm{methy} /$ prednisolone $500 \mathrm{mg}$, cisplatin 25 $\mathrm{mg} / \mathrm{m}^{2} / \mathrm{d}$, cytarabine $2000 \mathrm{mg} / \mathrm{m}^{2}$. FEC-100 = 5-fluorouracil $500 \mathrm{mg} / \mathrm{m}^{2}$, epirubicin $100 \mathrm{mg} / \mathrm{m}^{2}$, cyclophosphamide $500 \mathrm{mg} / \mathrm{m}^{2}$. FEC-high-dose = 5-fluorouracil $750 \mathrm{mg} / \mathrm{m}^{2}$, epirubicin $35 \mathrm{mg} / \mathrm{m}^{2}$, cyclophosphamide 400 $\mathrm{mg} / \mathrm{m}^{2}$. FOIL = 5-FU, oxaliplatin, irinotecan, leucovorin. FOLFIRI $=5$-FU, irinotecan, leucovorin. FOLFOX $=5$-FU, oxaliplatin, leucovorin. LNH- $87=$ cyclophosphamide $1200 \mathrm{mg} / \mathrm{m}^{2}$ day 1, vindesine $2 \mathrm{mg} / \mathrm{m}^{2}$ days $1 \& 5$, bleomycin $10 \mathrm{mg}$ days $1 \& 5$, prednisolone $60 \mathrm{mg} / \mathrm{m}^{2}$ days $1-5$, methotrexate $15 \mathrm{mg}$, with either doxorubicin $75 \mathrm{mg} / \mathrm{m}^{2}$ or mitoxantrone $12 \mathrm{mg} / \mathrm{m}^{2}$ day 1 . MAID = mesna, doxorubicin, ifosfamide, dacarbazine. RCHOP $=$ CHOP plus rituximab. TAC = doxorubicin $50 \mathrm{mg} / \mathrm{m}^{2}$, cyclophosphamide $500 \mathrm{mg} / \mathrm{m}^{2}$, docetaxel $75 \mathrm{mg} / \mathrm{m}^{2}$. VAPEC-B = vincristine $1.4 \mathrm{mg} / \mathrm{m}^{2}$, doxorubicin $35 \mathrm{mg} / \mathrm{m}^{2}$ prednisolone $50 \mathrm{mg} / \mathrm{d}$ (then tapered) etoposide $100 \mathrm{mg} / \mathrm{m}^{2}$, cyclophosphamide $350 \mathrm{mg} / \mathrm{m}^{2}$, bleomycin $10 \mathrm{mg} / \mathrm{m}^{2}$. VNCOP-B = vincristine $2 \mathrm{mg}$, mitoxantrone $10 \mathrm{mg} / \mathrm{m}^{2}$, cyclophosphamide $300 \mathrm{mg} / \mathrm{m}^{2}$, etoposide $150 \mathrm{mg} / \mathrm{m}^{2}$, prednisone $40 \mathrm{mg}$, bleomycin $10 \mathrm{mg} / \mathrm{m}^{2}$ 


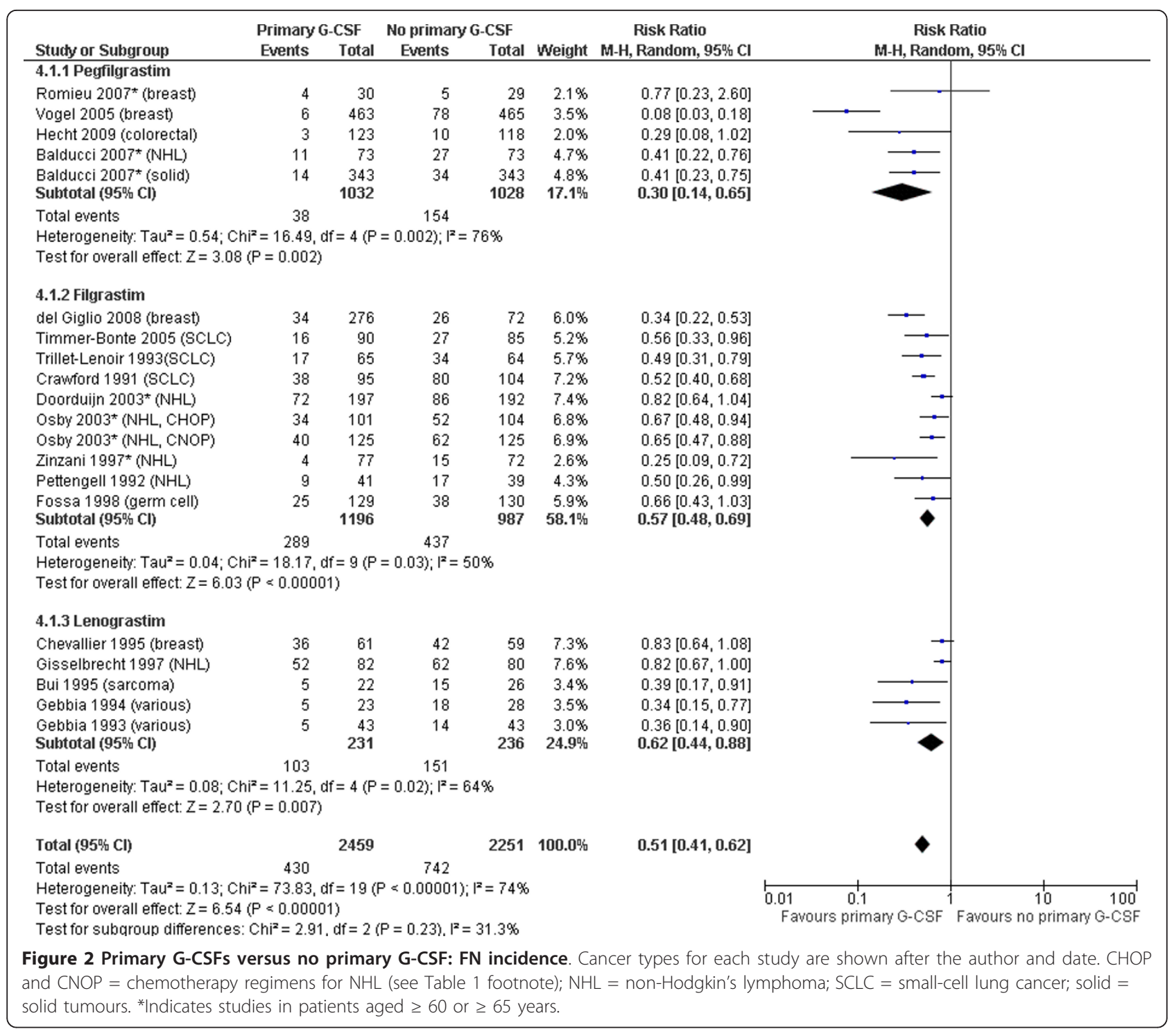

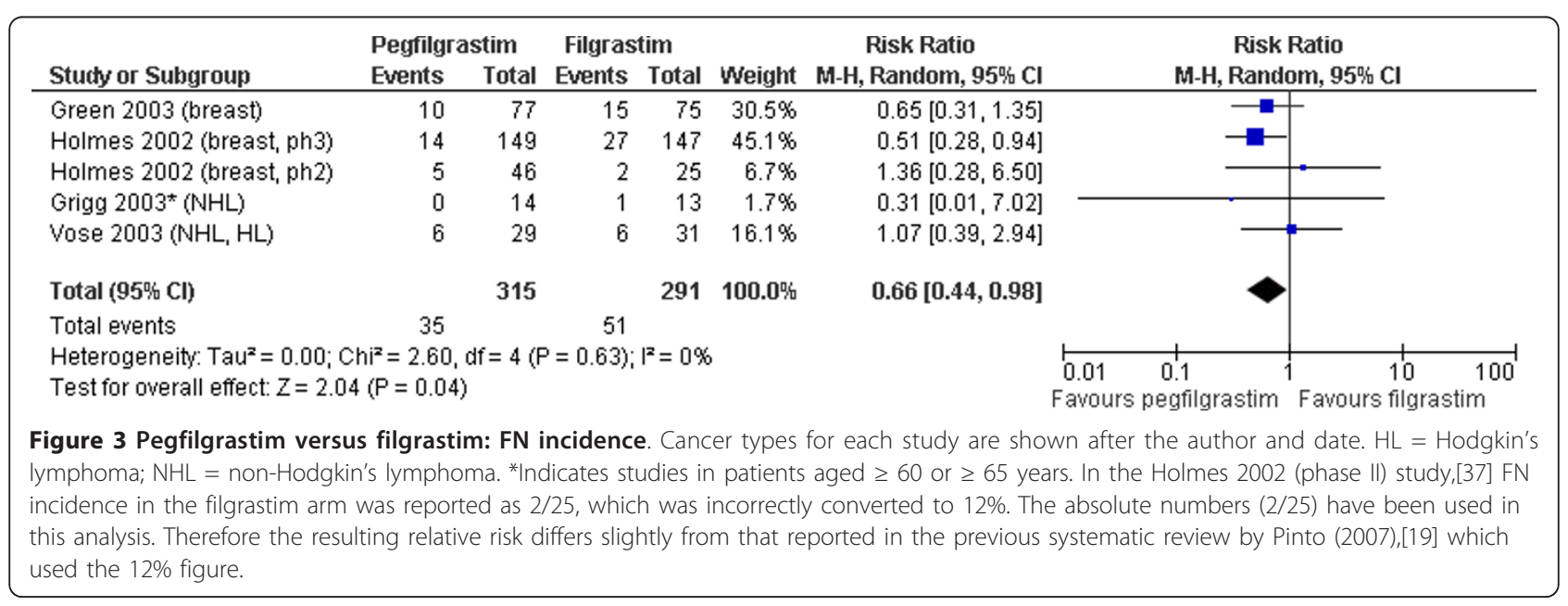


Table 2 Summary of febrile neutropenia incidence based on meta-analyses of trials of G-CSFs

\begin{tabular}{llcclll}
\hline Treatment $\mathbf{1}$ & Treatment $\mathbf{2}$ & No of studies & No of patients & Relative risk of FN (95\% Cl) & $\mathbf{p}$-value & $\mathbf{I}^{\mathbf{2}}$ (heterogeneity) \\
\hline Pegfilgrastim & No primary G-CSF & 5 & 2060 & $0.30(0.14$ to 0.65) & $p=0.002$ & $76 \%$ \\
\hline Filgrastim & No primary G-CSF & 10 & 2183 & $0.57(0.48$ to 0.69) & $p<0.00001$ & $50 \%$ \\
\hline Lenograstim & No primary G-CSF & 5 & 467 & $0.62(0.44$ to 0.88) & $p=0.007$ & $64 \%$ \\
\hline Any G-CSF & No primary G-CSF & 20 & 4710 & $0.51(0.41$ to 0.62) & $p<0.00001$ & $74 \%$ \\
\hline Pegfilgrastim & Filgrastim & 5 & 606 & $0.66(0.44$ to 0.98) & $p=0.04$ & $0 \%$ \\
\hline
\end{tabular}

In terms of comparisons between different G-CSFs, the relative risk of $\mathrm{FN}$ for pegfilgrastim versus filgrastim was 0.66 (95\% CI: 0.44 to 0.98 ). There were no head-tohead trials comparing lenograstim to either of the other two G-CSFs.

\section{Discussion}

Our systematic review and meta-analyses confirm and strengthen previous evidence that primary prophylaxis with each of the three G-CSFs is effective in reducing the risk of FN following chemotherapy. In particular, our systematic review identified 4 further RCTs of pegfilgrastim vs. no primary G-CSF, [22-24] whereas at the time of a previous systematic review [18] only a single RCT [21] making this comparison was available. Although these 5 RCTs comparing pegfilgrastim with no primary G-CSF were heterogeneous in terms of clinical population and chemotherapy regimen, the pooled relative risk indicated a significant effect of pegfilgrastim in reducing FN incidence. Filgrastim and lenograstim also significantly reduced FN incidence.

This review also strengthens the evidence base regarding the comparative effectiveness of the three G-CSFs; in particular, comparison of the "once-per-cycle" G-CSF pegfilgrastim versus the "once-daily" G-CSF filgrastim. Meta-analysis of five RCTs indicated that FN incidence was significantly lower following primary prophylaxis with pegfilgrastim than with filgrastim. This is consistent with the fact that the reduction in FN risk for pegfilgrastim versus no primary G-CSF was greater than the reduction observed for filgrastim versus no primary GCSF.

As discussed in previous reviews, $[18,19]$ there was heterogeneity among the studies in terms of the clinical population (age, cancer type), chemotherapy regimen, and cycle length and number. Correspondingly, heterogeneity was observed among the study results. Since individual studies differed on too many variables for formal sub-analyses to be meaningful, all studies were included in the analysis. There was no clear difference in G-CSF effectiveness between cancer types, nor in studies restricting to elderly populations. However, the variation in clinical population, and the corresponding high levels of heterogeneity, indicate that caution should be used when applying the results to individual clinical settings. Conversely, the range of populations and treatment regimens covered by the included studies is likely to reflect the variations which would be observed in clinical practice.

\section{Conclusions}

This systematic review and meta-analysis demonstrate that primary G-CSF prophylaxis with pegfilgrastim, filgrastim and lenograstim is effective in reducing the risk of FN in adults undergoing chemotherapy for solid tumours or lymphoma. In addition, although heterogeneity existed between studies, a meta-analysis suggests that pegfilgrastim reduces the risk of FN to a greater extent than filgrastim.

\section{Appendix 1: Search strategy (Medline)}

1 Granulocyte colony-stimulating factor/

2 Granulocyte colony-stimulating factor, recombinant/

3 Colony-stimulating factors, recombinant/

4. Filgrastim/

5 G-CSF\$

6 granulocyte colony-stimulating factor\$

7 filgrastim

8 Neupogen

9 pegfilgrastim

10 Neulasta

11 lenograstim

12 Granocyte

13 Euprotin

14. r-metHuG-CSF

15 SD-01

16 PEG-rmetHuG-CSF

17 XM02

18 Ratiograstim

19 or/1-18

20 randomized controlled trial.pt.

21 controlled clinical trial.pt.

22 randomized controlled trial/

23 random allocation/

24 double blind method/

25 single blind method/

26 clinical trial.pt.

27 exp clinical trial/ 
28 (clin\$ adj25 trial\$).ti,ab.

29 ( (singl\$ or doubl\$ or trebl\$ or tripl\$) adj25 (blind\$ or mask\$)).ti,ab.

30 placebos/

31 placebos.ti,ab.

32 random.ti,ab.

33 research design/

34 randomised.ti,ab

35 randomized.ti,ab

36 or $/ 20-35$

3719 and 36

("\$" indicates truncations; "/" indicates medical subject headings)

\section{List of abbreviations}

ANC: Absolute neutrophil count; ASCO: American Society of Clinical Oncology; DARE: Database of Abstracts of Reviews of Effects; EORTC: European Organisation for Research and Treatment of Cancer; FN: Febrile neutropenia; G-CSF: Granulocyte colony-stimulating factor; NCCN: National Comprehensive Cancer Network; NHS-EED: NHS Economic Evaluation Database; RCT: Randomised controlled trial.

\section{Acknowledgements and funding}

The research underlying this paper was funded by Amgen $L t d$, and a research grant from Amgen (EUROPE) $\mathrm{GmbH}$ was provided to support the production of the manuscript. Amgen staff reviewed and made suggested edits to the manuscript, but final content, authorship and right to publication remained with the research team.

\section{Author details}

'School of Health and Related Research (ScHARR), University of Sheffield, Sheffield, UK. ${ }^{2}$ Academic Unit of Primary Health Care, University of Bristol, Bristol, UK

\section{Authors' contributions}

KC undertook the systematic review and drafted the manuscript. JM undertook the statistical analyses. SW contributed to study selection and interpretation. MS contributed to study selection and interpretation and to the statistical analyses. RA participated in the design and coordination of the study and contributed to the statistical analyses. All authors read and approved the final manuscript.

\section{Competing interests}

The research underlying this paper was funded by Amgen $L t d$, and a research grant from Amgen (EUROPE) GmbH was provided to support the production of the manuscript. Amgen staff reviewed and made suggested edits to the manuscript, but final content, authorship and right to publication remained with the research team.

Received: 25 May 2011 Accepted: 23 September 2011 Published: 23 September 2011

\section{References}

1. Kuderer N, Dale D, Crawford J, Cosler L, Lyman G: Mortality, Morbidity, and Cost Associated with Febrile Neutropenia in Adult Cancer Patients. CANCER 2006, 106:2258-2266.

2. Brown R, Hutton J, Burrell A: Cost Effectiveness of Treatment Options in Advanced Breast Cancer in the UK. Pharmacoeconomics 2001, 19:1091-1102.

3. Brown RE, Hutton J: Cost-utility model comparing docetaxel and paclitaxel in advanced breast cancer patients. Anticancer Drugs 1998, 9.899-907.

4. Shayne M, Crawford J, Dale D, Culakova E, Lyman G, for the ANC Study Group: Predictors of reduced dose intensity in patients with early-stage breast cancer receiving adjuvant chemotherapy. Breast Cancer Res Treat 2006, 100:255-262
5. Bonadonna G, Moliterni A, Zambetti M, Daidone M, Pilotti S, Gianni L, et al: 30 years' follow up of randomised studies of adjuvant CMF in operable breast cancer: cohort study. BMJ 2005, 330:217-222.

6. Welte K, Gabrilove J, Bronchud MH, Platzer E, Morstyn G: Filgrastim ( $r$ metHuG-CSF): the first 10 years. Blood 1996, 88:1907-1929.

7. Green M, Koelbl H, Baselga J, GAlid A, Guillem V, Gascon P, et al: A randomized double-blind multicenter phase III study of fixed-dose single-administration pegfilgrastim versus daily filgrastim in patients receiving myelosuppressive chemotherapy. Annals of Oncology 2003, 14:29-35.

8. Holmes F, O'Shaughnessy J, Vukelja S, Jones S, Shogan J, Savin M, et al: Blinded, Randomized, Multicenter Study to Evaluate Single Administration Pegfilgrastim Once per Cycle Versus Daily Filgrastim as an Adjunct to Chemotherapy in Patients With High-Risk Stage II or Stage III/IV Breast Cancer. JOURNAL OF CLINICAL ONCOLOGY 2002, 20:727-731.

9. Chevallier $B$, Chollet $P$, Merrouche $Y$, Roche H, Fumoleau P, Kerbrat $P$, et al: Lenograstim prevents morbidity from intensive induction chemotherapy in the treatment of inflammatory breast cancer. J Clin Oncol 1995, 13:1564-1571.

10. Bui BN, Chevallier B, Chevreau C, Krakowski I, Peny AM, Thyss A, et al: Efficacy of lenograstim on hematologic tolerance to MAID chemotherapy in patients with advanced soft tissue sarcoma and consequences on treatment dose-intensity. J Clin Oncol 1995, 13:2629-2636.

11. Molineux G: The design and development of pegfilgrastim (PEGrmetHuG-CSF, Neulasta). Curr Pharm Des 2004, 10:1235-1244

12. Molineux G: Pegfilgrastim: using pegylation technology to improve neutropenia support in cancer patients. Anticancer Drugs 2003, 14:259-264.

13. Aapro MS, Bohlius J, Cameron DA, Dal LL, Donnelly JP, Kearney N, et al: 2010 update of EORTC guidelines for the use of granulocyte-colony stimulating factor to reduce the incidence of chemotherapy-induced febrile neutropenia in adult patients with lymphoproliferative disorders and solid tumours. Eur $J$ Cancer 2011, 47:8-32.

14. Smith TJ, Khatcheressian J, Lyman GH, Ozer H, Armitage JO, Balducci L, et al: 2006 update of recommendations for the use of white blood cell growth factors: an evidence-based clinical practice guideline. J Clin Oncol 2006, 24:3187-3205.

15. National Comprehensive Cancer Network (NCCN): Clinical Practice Guidelines in Oncology: Myeloid Growth Factors. 2011, 1-5-2010.

16. Moher D, Liberati A, Tetzlaff J, Altman DG: Preferred reporting items for systematic reviews and meta-analyses: the PRISMA statement. Ann Intern Med 2009, 151:264-269.

17. PRISMA statement website. 2009 [http://www.prisma-statement.org]

18. Kuderer N, Dale D, Crawford J, Lyman G: Impact of primary prophylaxis with granulocyte colony-stimulating factor on febrile neutropenia and mortality in adult cancer patients receiving chemotherapy: a systematic review. JOURNAL OF CLINICAL ONCOLOGY 2007, 25:3158-6731.

19. Pinto L, Liu Z, Doan Q: Comparison of pegfilgrastim with filgrastim on febrile neutropenia, grade IV neutropenia and bone pain: a metaanalysis of randomized controlled trials. Curr Med Res Opin 2007, 23:2283-2295.

20. The Cochrane Collaboration, Higgins JPT, Green S: Cochrane Handbook for Systematic Reviews of Interventions 2009.

21. Vogel C, Wojtukiewicz M, Carroll R, Tjulandin S, Barajas-Figueroa L, Wiens B, et al: First and Subsequent Cycle Use of Pegfilgrastim Prevents Febrile Neutropenia in Patients With Breast Cancer: A Multicenter, Double-Blind, Placebo-Controlled Phase III Study. J Clin Oncol 2005, 23:1178-1184.

22. Balducci L, Al-Halawani H, Charu V, Tam J, Shahin S, Dreiling L, et al: Elderly cancer patients receiving chemotherapy benefit from first-cycle pegfilgrastim. Oncologist 2007, 12:1416-1424.

23. Romieu G, Clemens M, Mahlberg R: Pegfilgrastim supports delivery of FEC-100 chemotherapy in elderly patients with high risk breast cancer: A randomized phase 2 trial. Critical Reviews in Oncology/Hematology 2007, 64:64-72.

24. Hecht JR, Pillai M, Gollard R, Dreiling L, Mo M, Malik I: Pegfilgrastim in colorectal cancer (CRC) patients (pts) receiving every-two-week (Q2W) chemotherapy (CT): Long-term results from a phase II, randomized, controlled study. JOURNAL OF CLINICAL ONCOLOGY 2009, 27:Abstract 4072. 
25. Doorduijn JK, van der HB, van Imhoff GW, van der Hem KG, Kramer MH, van Oers MH, et al: CHOP compared with CHOP plus granulocyte colonystimulating factor in elderly patients with aggressive non-Hodgkin's lymphoma. J Clin Oncol 2003, 21:3041-3050.

26. Osby E, Hagberg H, Kvaloy S, Teerenhovi L, Anderson H, Cavallin-Stahl E, et al: CHOP is superior to CNOP in elderly patients with aggressive lymphoma while outcome is unaffected by filgrastim treatment: results of a Nordic Lymphoma Group randomized trial. Blood 2003, 101:3840-3848.

27. Zinzani PL, Pavone E, Storti S, Moretti L, Fattori PP, Guardigni L, et al: Randomized trial with or without granulocyte colony-stimulating factor as adjunct to induction VNCOP-B treatment of elderly high-grade nonHodgkin's lymphoma. Blood 1997, 89:3974-3979.

28. Pettengell R, Gurney H, Radford J, Deakin D: Granulocyte ColonyStimulating Factor to Prevent Dose-Limiting Neutropenia in NonHodgkin's Lymphoma: A Randomized Controlled Trial. Blood 1992, 80:1430-1436.

29. Timmer-Bonte J, de Boo T, Smit H, Biesma B, Wilschut F, Cheragwandi S, et al: Prevention of Chemotherapy-Induced Febrile Neutropenia by Prophylactic Antibiotics Plus or Minus Granulocyte Colony-Stimulating Factor in Small-Cell Lung Cancer: A Dutch Randomized Phase III Study. J Clin Oncol 2005, 23:7974-7984.

30. Trillet-Lenoir V, Green J, Manegold C, Von Pawel J, Gatzemeier U, Lebeau B, et al: Recombinant granulocyte colony stimulating factor reduces the infectious complications of cytotoxic chemotherapy. European Journal of Cancer 1993, 29:319-324.

31. Crawford J, Ozer H, Stoller R, Johnson D, Lyman G, Tabbara I, et al: Reduction by granulocyte colony-stimulating factor of fever and neutropenia induced by chemotherapy in patients with small-cell lung cancer. N Engl J Med 1991, 325:164-170.

32. Fossa SD, Kaye SB, Mead GM, Cullen M, de WR, Bodrogi I, et al: Filgrastim during combination chemotherapy of patients with poor-prognosis metastatic germ cell malignancy. European Organization for Research and Treatment of Cancer, Genito-Urinary Group, and the Medical Research Council Testicular Cancer Working Party, Cambridge, United Kingdom. J Clin Oncol 1998, 16:716-724.

33. del Giglio A, Eniu A, Ganea-Motan D, Topuzov E, Lubenau H: XM02 is superior to placebo and equivalent to Neupogen in reducing the duration of severe neutropenia and the incidence of febrile neutropenia in cycle 1 in breast cancer patients receiving docetaxel/doxorubicin chemotherapy. BMC Cancer 2008, 8:332.

34. Gisselbrecht C, Haioun C, Lepage E, Bastion Y, Tilly H, Bosly A, et al: Placebo-controlled phase III study of lenograstim (glycosylated recombinant human granulocyte colony-stimulating factor) in aggressive non-Hodgkin's lymphoma: factors influencing chemotherapy administration. Groupe d'Etude des Lymphomes de l'Adulte. Leuk Lymphoma 1997, 25:289-300.

35. Gebbia V, Valenza R, Testa A, Cannata G, Borsellino N, Gebbia N: A prospective randomized trial of thymopentin versus granulocyte-colony stimulating factor with or without thymopentin in the prevention of febrile episodes in cancer patients undergoing highly cytotoxic chemotherapy. Anticancer Res 1994, 14:731-734.

36. Gebbia V, Testa A, Valenza R, Borsellino N, Cipolla C, Cannata G, et al: A prospective evaluation of the activity of human granulocyte-colony stimulating factor on the prevention of chemotherapy-related neutropenia in patients with advanced carcinoma. J Chemother 1993, 5:186-190

37. Holmes F, Jones S, O'Shaughnessy J, Vukelja S, George T, Savin M, et al: Comparable efficacy and safety profiles of once-per-cycle pegfilgrastim and daily injection filgrastim in chemotherapy-induced neutropenia: a multicenter dose-finding study in women with breast cancer. Annals of Oncology 2002, 13:903-909.

38. Grigg A, Solal-Celigny P, Hoskin P, Taylor K, McMillan A, Forstpointner R, et al: Open-label, randomized study of pegfilgrastim vs. daily filgrastim as an adjunct to chemotherapy in elderly patients with non-Hodgkin's lymphoma. Leuk Lymphoma 2003, 44:1503-1508.

39. Vose J, Crump M, Lazarus H, Emmanouilides C, Schenkein D, Moore J, et al: Randomized, Multicenter, Open-Label Study of Pegfilgrastim Compared With Daily Filgrastim After Chemotherapy for Lymphoma. JOURNAL OF CLINICAL ONCOLOGY 2003, 21:514-519.

\section{Pre-publication history}

The pre-publication history for this paper can be accessed here: http://www.biomedcentral.com/1471-2407/11/404/prepub

doi:10.1186/1471-2407-11-404

Cite this article as: Cooper et al:: Granulocyte colony-stimulating factors for febrile neutropenia prophylaxis following chemotherapy: systematic review and meta-analysis. BMC Cancer 2011 11:404.

\section{Submit your next manuscript to BioMed Central and take full advantage of:}

- Convenient online submission

- Thorough peer review

- No space constraints or color figure charges

- Immediate publication on acceptance

- Inclusion in PubMed, CAS, Scopus and Google Scholar

- Research which is freely available for redistribution 